\title{
AGILE: a seamless phase I/lla platform for the rapid evaluation of candidates for COVID-19 treatment: an update to the structured summary of a study protocol for a randomised platform trial letter
}

\author{
Gareth O. Griffiths ${ }^{1 *}$, Richard FitzGerald ${ }^{2}$, Thomas Jaki ${ }^{3}$, Andrea Corkhill', Helen Reynolds ${ }^{4}$, Sean Ewings ${ }^{1}$, \\ Susannah Condie', Emma Tilt', Lucy Johnson' ${ }^{1}$, Mike Radford', Catherine Simpson', Geoffrey Saunders ${ }^{1}$, \\ Sara Yeats ${ }^{1}$, Pavel Mozgunov ${ }^{3}$, Olana Tansley-Hancock ${ }^{1}$, Karen Martin ${ }^{1}$, Nichola Downs ${ }^{1}$, Izabela Eberhart ${ }^{1}$, \\ Jonathan W. B. Martin ${ }^{1}$, Cristiana Goncalves ${ }^{1}$, Anna Song ${ }^{1}$, Tom Fletcher ${ }^{5}$, Kelly Byrne ${ }^{5}$, David G. Lalloo ${ }^{5}$, \\ Andrew Owen ${ }^{4}$, Michael Jacobs ${ }^{6}$, Lauren Walker², Rebecca Lyon², Christie Woods' ${ }^{2}$ Jennifer Gibney², \\ Justin Chiong ${ }^{2,4}$, Nomathemba Chandiwana ${ }^{7}$, Shevin Jacob ${ }^{5}$, Mohammed Lamorde ${ }^{8}$, Catherine Orrell ${ }^{9}$, \\ Munir Pirmohamed ${ }^{2,4}$, Saye Khoo ${ }^{2,4}$ and on behalf of the AGILE investigators
}

\begin{abstract}
Background: There is an urgent unmet clinical need for the identification of novel therapeutics for the treatment of COVID-19. A number of COVID-19 late phase trial platforms have been developed to investigate (often repurposed) drugs both in the UK and globally (e.g. RECOVERY led by the University of Oxford and SOLIDARITY led by $\mathrm{WHO}$ ). There is a pressing need to investigate novel candidates within early phase trial platforms, from which promising candidates can feed into established later phase platforms. AGILE grew from a UK-wide collaboration to undertake early stage clinical evaluation of candidates for SARS-CoV-2 infection to accelerate national and global healthcare interventions.

Methods/design: AGILE is a seamless phase I/lla platform study to establish the optimum dose, determine the activity and safety of each candidate and recommend whether it should be evaluated further. Each candidate is evaluated in its own trial, either as an open label single arm healthy volunteer study or in patients, randomising between candidate and control usually in a 2:1 allocation in favour of the candidate. Each dose is assessed sequentially for safety usually in cohorts of 6 patients. Once a phase II dose has been identified, efficacy is assessed by seamlessly expanding into a larger cohort. AGILE is completely flexible in that the core design in the master protocol can be adapted for each candidate based on prior knowledge of the candidate (i.e. population, primary endpoint and sample size can be amended). This information is detailed in each candidate specific trial protocol of
\end{abstract}

\footnotetext{
*Correspondence: G.O.Griffiths@soton.ac.uk

'Southampton Clinical Trials Unit, University of Southampton, Southampton, Hampshire, UK

Full list of author information is available at the end of the article
}

\section{$\triangle B M C$}

(c) The Author(s). 2021 Open Access This article is licensed under a Creative Commons Attribution 4.0 International License, which permits use, sharing, adaptation, distribution and reproduction in any medium or format, as long as you give appropriate credit to the original author(s) and the source, provide a link to the Creative Commons licence, and indicate if changes were made. The images or other third party material in this article are included in the article's Creative Commons licence, unless indicated otherwise in a credit line to the material. If material is not included in the article's Creative Commons licence and your intended use is not permitted by statutory regulation or exceeds the permitted use, you will need to obtain permission directly from the copyright holder. To view a copy of this licence, visit http://creativecommons.org/licenses/by/4.0/ The Creative Commons Public Domain Dedication waiver (http://creativecommons.org/publicdomain/zero/1.0/) applies to the data made available in this article, unless otherwise stated in a credit line to the data. 
the master protocol.

Discussion: Few approved treatments for COVID-19 are available such as dexamethasone, remdesivir and tocilizumab in hospitalised patients. The AGILE platform aims to rapidly identify new efficacious and safe treatments to help end the current global COVID-19 pandemic. We currently have three candidate specific trials within this platform study that are open to recruitment.

Trial registration: EudraCT Number: 2020-001860-27 14 March 2020

ClinicalTrials.gov Identifier: NCT04746183 19 February 2021

ISRCTN reference: 27106947

Keywords: COVID-19, SARS-CoV-2, Randomised controlled trial, Platform study, Master protocol, Phase I/II, Bayesian

\section{Background}

In 2019, a novel coronavirus disease (COVID-19) emerged in Wuhan, China, which is caused by severe acute respiratory syndrome coronavirus 2 (SARS-CoV-2) [1]. Many patients do not progress to severe disease, but as COVID-19 spread across the world, vast numbers of patients have been hospitalised with respiratory failure, a significant proportion of whom have become critically unwell and died [2-4]. The progression from initial symptoms, most commonly fever, fatigue and cough, to respiratory failure requiring oxygen support or mechanical ventilation occurs several days after the onset of symptoms [2]. While treatment options such as dexamethasone, remdesivir and tocilizumab are now available for certain hospitalised patients with severe disease, these are repurposed medicines [5, 6]. AGILE is unique as it provides a platform for assessing novel therapeutic agents. There is also a distinct lack of available treatment options for the early phase of infection which may help prevent progression to severe disease [7].

Given the rapid and global spread of COVID-19 and the emergence of new variants in the UK, South Africa, India and Brazil, a robust but rapid assessment of potential treatments is needed [8]. The AGILE platform aims to identify new candidates with promising evidence of efficacy that can be further evaluated in subsequent larger phase IIb/III trials. Having one master protocol ensures different candidates are evaluated in the same consistent manner and enables more efficient introduction of new trials for new candidates.

\section{Methods/design Objectives}

Phase I-To determine the optimal dose of each candidate (or combination of candidates) entered into the platform. Phase II-To determine the efficacy and safety of each candidate entered into the platform, compared to the current Standard of Care (SoC), and recommend whether it should be evaluated further in later phase II and III platforms.

\section{Trial design}

AGILE is a multicentre, multi-candidate, multi-dose, multi-stage, randomised phase I/IIa Bayesian adaptive platform trial to determine the safety and efficacy of multiple candidate agents for the treatment of COVID19. The multi-candidate design allows many candidates to be tested simultaneously (while potentially sharing control group data, provided they are at least contemporaneously recruited), in order to increase efficiency compared to multiple single-candidate studies. The multi-dose feature allows progression beyond the licensed dose dependent on adequate safety and tolerability data and promising efficacy. The multi-stage feature allows for pre-specified analyses that can be used to determine dropping of ineffective doses or candidates or recommending doses or candidates for further phase II/ III testing, further increasing efficiency of the study. The adaptive platform design allows for the removal of unpromising candidates, promising candidates to be recommended for further testing in external phase II/III studies, and the addition of new potential treatments to be added during the trial. Candidates will be added into the AGILE platform study via candidate specific trial (CST) protocols to the master protocol.

\section{Participants}

Patient populations can vary between CSTs, but the main eligibility criteria include adult patients ( $\geq 18$ years) who have laboratory-confirmed infection with SARS$\mathrm{CoV}-2$. We will include both severe and mild-moderate patients as well as healthy volunteers defined as follows: group A (severe disease)-patients with WHO Clinical Progression Scale [9] of grades 5 (hospitalised, oxygen by mask or nasal prongs), 6 (hospitalised, non-invasive ventilation or high flow oxygen), 7 [hospitalised, intubation and mechanical ventilation, arterial oxygen partial pressure $\left(\mathrm{PaO}_{2}\right) /$ fractional inspired oxygen $\left(\mathrm{FiO}_{2}\right) \geq 150$ or peripheral capillary oxygen saturation $\left(\mathrm{SpO}_{2}\right) / \mathrm{FiO}_{2} \geq$ 200], 8 [hospitalised mechanical ventilation $\mathrm{PaO}_{2} / \mathrm{FiO}_{2}<$ $150\left(\mathrm{SpO}_{2} / \mathrm{FiO}_{2}<200\right)$ or vasopressors] or 9 (hospitalised, mechanical ventilation $\mathrm{pO}_{2} / \mathrm{FiO}_{2}<150$ and 
vasopressors, dialysis or ECMO). Group B (mild-moderate disease)-ambulant or hospitalised patients with $\mathrm{SpO}_{2}>94 \%$ room air. If any CSTs include participants from the community setting, the CST protocol will clarify whether patients with suspected SARS-CoV-2 infection (in addition to laboratory-confirmed infection) are also eligible. Group C (healthy volunteers) - to be defined in each CST protocol. Participants are currently being recruited from the UK, but we intend to open up recruitment at international sites later in 2021.

\section{Intervention and comparator}

The comparator is the current SoC, and in some CSTs, placebo is added to the current SoC. Candidates that prevent uncontrolled cytokine release, prevention of viral replication, and other anti-viral treatment strategies are at various stages of development for inclusion into AGILE. Other CSTs will be added over time. There is not a set limit on the number of CSTs we can include within the AGILE master protocol, and we will upload each CST into this publication as each opens to recruitment.

\section{Main outcomes}

Phase I-Dose limiting toxicities using Common Terminology Criteria for Adverse Events (CTCAE) Version 5 Grade $\geq 3$ adverse events. Phase II-Agreed on a CST basis depending on mechanism of action of the candidate and patient population, but may include time to clinical improvement of at least 2 points on the WHO Clinical Progression Scale [measured up to 29 days from randomisation], progression of disease $\left(\mathrm{SpO}_{2}<92 \%\right)$ or hospitalisation or death, or change in time-weighted viral load [measured up to 29 days from randomisation].

\section{Randomisation and blinding}

Each candidate will be evaluated in its own CST, either as an open label single arm healthy volunteer study or in patients, randomising between candidate and control. Randomisation varies with each CST, but the default is 2:1 allocation in favour of the candidate to maximise early safety data. Each dose will be assessed sequentially for safety in cohorts of patients; the default size is 6 patients per cohort. Once a phase II dose has been identified, we will assess efficacy by seamlessly expanding into a larger cohort.

\section{Numbers to be randomised (sample size)}

Sample size varies between CSTs. Simulations have shown that around 16 participants are necessary to determine futility or promise of a candidate at a given dose (in efficacy evaluation alone) and between 32 and 40 participants are required across the dose-finding and efficacy evaluation when capping the maximum number of participants contributing to the evaluation of a treatment at 40. Each CST protocol will give details on the sample size needed for that candidate and a justification for any CST needing more than 40 participants.

\section{Full protocol}

The full AGILE master protocol is attached as an additional file, accessible from the Trials website www. agiletrial.net (Additional file 1). In the interest of expediting dissemination of this material, the familiar formatting has been eliminated; this addendum is an update to the original letter and serves as a summary of the key elements of the full master protocol and trial status of active CSTs.

\section{Discussion}

AGILE is the first and only phase I/IIa national clinical trial platform in the UK to determine the safety and efficacy of promising candidate agents for COVID-19. It bridges the gap between preclinical development and later phase trials, so that potential new treatments can pass through the phases of drug development in a rapid and safe manner. An independent COVID-19 Therapeutics Advisory Panel (UK-CTAP) will evaluate and prioritise candidate agents for inclusion in the study. The design of AGILE allows for the assessment of multiple candidates at different doses, with the ability to add candidates as they are identified or drop them as their evaluation is completed. Promising candidates will move to an external trial for further evaluation in the phase II/ III setting. AGILE is completely flexible and each CST design is catered to the requirement of the candidate whereby population, primary endpoint and sample size can be amended. AGILE has full regulatory approval [by the UK MHRA, ethics and HRA and the WHO Research Ethics Review Committee (ERC)] and has three CSTs currently open to recruitment with a number of others at various stages of development. AGILE has the potential to rewrite the treatment landscape for COVID-19 and shape our response to future global pandemics.

\section{Trial status}

\section{AGILE master protocol}

The AGILE master protocol Version 9 (17 February 2021) has full regulatory approval and utilises several digital technology solutions, including Medidata's Rave Electronic Data Capture (EDC), Randomization and Trial Supply Management (RTSM) for randomisation and drug distribution, patient eConsent on iPads and electronic Clinical Outcome Assessment (eCOA) to collect patient reported outcome measures via an App on the participants smart phones. 
CST-2: a randomised, multicentre, seamless, adaptive, phase $\mathrm{I} / \mathrm{II}$ platform study to determine the optimal dose, safety and efficacy of Molnupiravir (EIDD-2801) for the treatment of COVID-19

CST-2 has full regulatory approval, has completed phase I and has successfully determined a dose for phase II evaluation. Phase II recruitment is ongoing following a planned interim analysis by an Independent Data Monitoring Committee. Molnupiravir is being evaluated in adult ambulant patients with laboratory confirmed COVID-19, who are within 5 days of symptom onset in the UK. Molnupiravir (EIDD-2801) is the $5^{\prime}$-isopropyl ester prodrug of the broadly active, direct-acting antiviral ribonucleoside analogue EIDD-1931. Molnupiravir is being developed for the treatment of infections caused by RNA viruses, specifically for COVID-19 and other coronavirus infections, influenza and Venezuelan equine encephalitis virus $[10,11]$.

CST-3A: a multicentre, adaptive, phase I trial to determine the optimal dose, safety and efficacy of nitazoxanide for the treatment of COVID-19

CST-3A has full regulatory approval and has recently completed recruitment. Nitazoxanide is being evaluated in healthy adults in the UK. Nitazoxanide is a thiazolide FDA-approved antiparasitic medicine used for the treatment of cryptosporidiosis and giardiasis diarrhoea but has been studied previously for viruses including influenza [12]. However, preclinical candidate evaluation by AGILE investigators using data from in vitro antiviral activity and historical human pharmacokinetic studies strongly suggests that doses higher than those approved will be required for durable antiviral activity in SARSCoV-2 infection [13, 14]. N.B. Once CST-3A has completed in the UK, it is planned that a CST-3B phase Ib/II trial will open in South Africa and Uganda in adult ambulant patients with laboratory-confirmed COVID-19.

CST-5: a randomised, multicentre, seamless, adaptive, phase I/II platform study to determine the phase II dose of VIR-7832, and evaluate the safety and efficacy of VIR7831 and VIR-7832 for the treatment of COVID-19

CST-5 has full regulatory approval and phase I is currently recruiting. VIR-7832 and VIR-7831 are being evaluated in adult ambulant patients with laboratoryconfirmed COVID-19, who are within 5 days of symptom onset in the UK. VIR-7832 and VIR-7831 are both human monoclonal antibodies that neutralise COVID19 by targeting the receptor-binding domain of the SARS-CoV-2 Spike (S) glycoprotein [15]. The binding of this domain inhibits the entry of the SARS-CoV-2 virus into the host cell. VIR-7832 includes the "XX2" modification in the fragment crystallisable domain. The XX2 modification is designed to enhance effector functions, abrogate $\mathrm{C} 1 \mathrm{q}$ binding and elicit enhanced $\mathrm{T}$ cell and antibody responses [16].

\section{Abbreviations}

COVID-19: Coronavirus disease 2019; CST: Candidate specific trial; CTCAE v5: Common Terminology Criteria for Adverse Events Version 5; eCOA: Electronic Clinical Outcome Assessment; EDC: Electronic Data Capture; ERC: Ethics Review Committee; $\mathrm{FiO}_{2}$ : Fractional inspired oxygen; $\mathrm{PaO}_{2}$ : Arteria oxygen partial pressure; RTSM: Randomization and Trial Supply Management; SARS-CoV-2: Severe acute respiratory syndrome coronavirus 2; SoC: Standard of Care; $\mathrm{SpO}_{2}$ : Peripheral capillary oxygen saturation; UK-CTAP: UK COVID-19 Therapeutics Advisory Panel

\section{Supplementary Information}

The online version contains supplementary material available at https://doi. org/10.1186/s13063-021-05458-4

Additional file 1.. AGILE Master Protocol Version 9 17-Feb-2021

\section{Acknowledgements}

We acknowledge Cancer Research UK (CRUK) who, during the COVID-19 crisis, allowed its staff at Southampton CTU to work with the National Institute for Health Research (NIHR) community (e.g. Southampton CTU, Liverpool CRF \& Southampton Biomedical Research (entre) to develop this phase I/II trial platform. We acknowledge the University Hospital Southampton NHS Foundation Trust, Liverpool University Hospitals NHS Foundation Trust, University of Southampton and the University of Liverpool who supported and collaborated in the development of AGILE. We acknowledge the Global Health Trials Unit within the Liverpool School of Tropical Medicine who are leading in developing AGILE international trials with our international partners in South Africa and Uganda. We also acknowledge The Wellcome Trust and the Medical Research Council (MRC)/UK Research and Innovation (UKRI) for core funding this project.

\section{Authors' contributions}

GG, SK and RF came up with the concept of the development of AGILE and oversaw the development of the master protocol and this submission. TJ and PM were responsible for the development of the statistical design. SE and GS were responsible for the statistical aspects of running and analysing the trial. $A C, H R$, LW, RL, CW, AS and JG were responsible for developing the master trial management and delivery aspects of the trial. SC, ET, $L$ and MR were responsible for the development of data collection and management aspects of the trial. SY was responsible for quality assurance and pharmacovigilance aspects of the study. OTH, KM, CS, ND, IE, JM, CG and KB were responsible for development of the CST protocols and submission. AO led pre-trial evaluation of the candidacy for CST3A. TF, LW, RF, AO, MJ, DL, SK and GG undertook candidate-specific protocol development for UK CSTs along with KB, NC, SJ, ML and CO for international CSTs. JC was responsible for managing the financial aspects of AGILE. SK and AO designed and reviewed the pharmacokinetic assessments within candidate-specific trials. All were involved in the development of the updated master protocol and this submission. The author(s) read and approved the final manuscript.

\section{Authors' information}

GG (director of the NIHR/CRUK Southampton Clinical Trials Unit), RF (director of the NIHR Clinical Research Facility (CRF) in Liverpool) and SK (chief investigator of AGILE) have developed this master protocol for, and on behalf of the UK NIHR and CRF community and COVID-19 patients. We are currently running AGILE in the UK but are working with international sites in low and middle-income countries to set up AGILE as an international trial platform.

\section{Funding}

The development of the AGILE master protocol, electronic data capture system, randomisation and eConsent was funded using Cancer Research UK (CRUK) core funding and National Institute for Health Research (NIHR) CTU support funding at the Southampton Clinical Trials Unit. NIHR funding at the Royal Liverpool and Broadgreen Clinical Research Facility and staff at the University of Liverpool and Lancaster also supported the development of the 
master protocol and its associated documentation (e.g. participant information sheets, case report forms). Funding for each CST protocol is a mixture of core funding and investigator-initiated research grants from pharmaceutical companies supplying the candidate. Funding for CST3 was provided by Unitaid and preclinical evaluation of nitazoxanide was supported by UKRI using funding repositioned from EP/R024804/1 as part of the UK emergency response to COVID-19. We have core funding from The Wellcome Trust and the Medical Research Council (MRC)/UK Research and Innovation (UKRI). TJ was supported by the NIHR, Cambridge Biomedical Research Centre (BRC-1215-20014) and by UK Medical Research Council (grant number: MC_UU_00002/14). PM was supported by NIHR Advanced Fellowship (NIHR300576).The views expressed are those of the author(s) and not necessarily those of the NIHR or the Department of Health and Social Care.

\section{Availability of data and materials}

When the database from each CST protocol is locked, analysed and published, we will make the data available to the academic community via a request to the AGILE Data Sharing Committee (agile@soton.ac.uk).

\section{Declarations}

\section{Ethics approval and consent to participate}

West Midlands - Edgbaston Research Ethics Committee (REC reference: 20/ WM/0136), 12/05/2020, https://www.hra.nhs.uk/planning-andimprovingresearch/application-summaries/research-summaries/agileaccordaccelerating-covid-19-drug-development-covid-19-uph/, I certify that this trial has received appropriate ethical approval as described above. We will obtain informed consent from all participants entering into AGILE. If the patient lacks capacity to give consent due to the severity of their medical condition (e.g. patients with WHO Clinical Progression Scale of Grades 7 (hospitalised, intubation and mechanical ventilation, $\mathrm{PaO}_{2} / \mathrm{FiO}_{2} \geq 150$ or $\mathrm{SpO}_{2} / \mathrm{FiO}_{2} \geq 200$ ), 8 (hospitalised mechanical ventilation $\mathrm{PaO}_{2} / \mathrm{FiO}_{2}<150$ $\left(\mathrm{SpO}_{2} / \mathrm{FiO}_{2}<200\right)$ or vasopressors or 9 (hospitalised, mechanical ventilation $\mathrm{PaO}_{2} / \mathrm{FiO}_{2}<150$ and vasopressors, dialysis or ECMO), then in the first instance, consent may be obtained from the patient's personal legal representative.

\section{Consent for publication}

Not applicable.

\section{Competing interests}

$\mathrm{AO}$ is a Director of Tandem Nano Ltd and co-inventor of patents relating to drug delivery. Also unrelated, $\mathrm{AO}$ has received research funding from ViiV, Merck, Janssen and consultancy from Gilead, ViiV and Merck. MP receives research funding from various organisations including the MRC and NIHR. He has also received partnership funding for the following: MRC Clinical Pharmacology Training Scheme (co-funded by MRC and Roche, UCB, Eli Lilly and Novartis), a PhD studentship jointly funded by EPSRC and Astra Zeneca and grant funding from Vistagen Therapeutics. He has also unrestricted educational grant support for the UK Pharmacogenetics and Stratified Medicine Network from Bristol-Myers Squibb and UCB. He has developed an HLA genotyping panel with MC Diagnostics, but does not benefit financially from this. He is part of the IMI Consortium ARDAT (www.ardat.org). None of these of funding sources have been used for the current paper.

\section{Author details}

${ }^{1}$ Southampton Clinical Trials Unit, University of Southampton, Southampton, Hampshire, UK. ${ }^{2}$ NIHR Royal Liverpool and Broadgreen CRF, Liverpool University Hospitals NHS Foundation Trust, Liverpool, UK. ${ }^{3}$ Lancaster University, Lancaster UK and MRC Biostatistics Unit, University of Cambridge, Cambridge, UK. ${ }^{4}$ University of Liverpool, Liverpool, UK. ${ }^{5}$ Liverpool School of Tropical Medicine, Liverpool, UK. ${ }^{6}$ Royal Free London NHS Foundation Trust, London, UK. ${ }^{7}$ University of the Witwatersrand, Johannesburg, South Africa. ${ }^{8}$ Infectious Diseases Institute, Makerere University, Kampala, Uganda. ${ }^{9}$ Desmond Tutu Health Foundation, University of Cape Town, Cape Town, South Africa.
Received: 29 March 2021 Accepted: 14 July 2021

Published online: 26 July 2021

\section{References}

1. Zhu N, Zhang D, Wang W, Li X, Yang B, Song J, et al. A novel coronavirus from patients with pneumonia in China, 2019. N Engl J Med. 2020;382(8): 727-33. https://doi.org/10.1056/NEJMoa2001017.

2. Huang C, Wang Y, Li X, Ren L, Zhao J, Hu Y, et al. Clinical features of patients infected with 2019 novel coronavirus in Wuhan, China. Lancet. 2020;395(10223):497-506. https://doi.org/10.1016/S0140-6736(20)30183-5.

3. Chen N, Zhou M, Dong X, Qu J, Gong F, Han Y, et al. Epidemiological and clinical characteristics of 99 cases of 2019 novel coronavirus pneumonia in Wuhan, China: a descriptive study. Lancet. 2020;395(10223):507-13. https:// doi.org/10.1016/S0140-6736(20)30211-7.

4. Wang D, Hu B, Hu C, Zhu F, Liu X, Zhang J, et al. Clinical characteristics of 138 hospitalized patients with 2019 novel coronavirus-infected pneumonia in Wuhan, China. Jama. 2020;323(11):1061-9. https://doi.org/10.1001/jama.2 020.1585 .

5. European Medicines Agency: Treatments and vaccines for Covid 19: authorised medicines 2021. https://www.ema.europa.eu/en/human-regula tory/overview/public-health-threats/coronavirus-disease-covid-19/trea tments-vaccines-covid-19.

6. Wise J. Covid-19: Arthritis drug tocilizumab reduces deaths in hospitalised patients, study shows. Bmj. 2021;372:n433.

7. Kim PS, Read SW, Fauci AS. Therapy for early COVID-19: a critical need Jama. 2020;324(21):2149-50. https://doi.org/10.1001/jama.2020.22813.

8. Burki T. Understanding variants of SARS-CoV-2. The Lancet. 2021;397(10273): 462. https://doi.org/10.1016/S0140-6736(21)00298-1.

9. WHO Working Group on the Clinical Characterisation and Management of COVID-19 infection. A minimal common outcome measure set for COVID19 clinical research. Lancet Infect Dis. 2020;20(8):e192-e7.

10. Agostini ML, Pruijssers AJ, Chappell JD, Gribble J, Lu X, Andres EL, et al. Small-molecule antiviral $\beta$-d-N (4)-hydroxycytidine inhibits a proofreadingintact coronavirus with a high genetic barrier to resistance. J Virol. 2019; 93(24):e01348-19. https://doi.org/10.1128/JVI.01348-19.

11. Toots M, Yoon JJ, Cox RM, Hart M, Sticher ZM, Makhsous N, et al. Characterization of orally efficacious influenza drug with high resistance barrier in ferrets and human airway epithelia. Sci Transl Med. 2019;11(515): eaax5866. https://doi.org/10.1126/scitransImed.aax5866.

12. Haffizulla J, Hartman A, Hoppers M, Resnick H, Samudrala S, Ginocchio C, et al. Effect of nitazoxanide in adults and adolescents with acute uncomplicated influenza: a double-blind, randomised, placebocontrolled, phase 2b/3 trial. Lancet Infect Dis. 2014;14(7):609-18. https://doi.org/10.101 6/S1473-3099(14)70717-0.

13. Arshad U, Pertinez H, Box H, Tatham L, Rajoli RKR, Curley P, et al. Prioritization of anti-SARS-Cov-2 drug repurposing opportunities based on plasma and target site concentrations derived from their established human pharmacokinetics. Clin Pharmacol Ther. 2020;108(4):775-90. https://doi.org/1 0.1002/cpt.1909.

14. Rajoli RKR, Pertinez H, Arshad U, Box H, Tatham L, Curley P, et al. Dose prediction for repurposing nitazoxanide in SARS-CoV-2 treatment or chemoprophylaxis. Br J Clin Pharmacol. 2021;87(4):2078-88. https://doi.org/1 $0.1111 /$ bcp. 14619

15. Pinto D, Park YJ, Beltramello M, Walls AC, Tortorici MA, Bianchi S, et al. Cross-neutralization of SARS-COV-2 by a human monoclonal SARS-CoV antibody. Nature. 2020;583(7815):290-5. https://doi.org/10.1038/s41586-02 0-2349-y.

16. Bournazos S, Corti D, Virgin HW, Ravetch JV. Fc-optimized antibodies elicit CD8 immunity to viral respiratory infection. Nature. 2020;588(7838):485-90. https://doi.org/10.1038/s41586-020-2838-z.

\section{Publisher's Note}

Springer Nature remains neutral with regard to jurisdictional claims in published maps and institutional affiliations. 